\title{
Variability in the Measurement of Human Ventricular Refractoriness
}

\author{
ALAN H. KADISH, STEPHEN SCHMALTZ, and FRED MORADY \\ From the Division of Cardiology and the Clinical Research Center, Department of Internal \\ Medicine, University of Michigan Medical Center, Ann Arbor, Michigan
}

KADISH, A.H., ET AL.: Variability in the Measurement of Human Ventricular Refractoriness. The degree of variability in ventricular refractoriness and factors potentially affecting this variability were evaluated in 80 patients undergoing an electrophysiological study. Each of seven variables (stimulation current, coupling interval of the basic drive train to spontaneous rhythm, pause between determinations, bipolar pacing configuration, bipolar vs unipolar pacing, atrioventricular synchrony, and autonomic tone) was evaluated in a group of ten patients to determine its effects on the reproducibility of refractoriness. Measurements were repeated ten times in every patient under each of two conditions. Five variables had significant effects on the reproducibility of measurements. Pacing at $10 \mathrm{~mA}$ was associated with less variability in the determination of ventricular refractoriness than pacing at twice threshold (within-subject variance component $4.5 \mathrm{vs} 10.1 \mathrm{msec} ; \mathrm{P}<0.001$ ). The mean difference between the longest and shortest determinations of refractory periods (range) was $6.2 \mathrm{msec}$ at $10 \mathrm{~mA}$ and $8.6 \mathrm{msec}$ at twice threshold. The use of a conditioning period of pacing and continuous trains (eight beats with a 3-sec pause) rather than a variable pause between serial trials reduced the mean within-subject variance component from 16.5 to $3.3(P<0.001)$ and the mean range of refractory period determinations from 10.8 to 4.8. The use of the distal rather than the proximal pole as the cathode decreased the mean withinsubject variance component from 9.4 to $3.3(P<0.001)$ and the range of determinations from 6.4 to 5.8 msec. Unipolar pacing was associated with less variability than bipolar pacing (mean within-subject variance component 4.6 vs $6.4 ; P<0.05$, mean range 5.0 vs $7.6 \mathrm{msec}$ ). In patients with ventriculoatrial dissociation, atrioventricular simultaneous pacing during the basic drive train decreased the withinsubject variance component from 21.2 to $5.7(P<0.001)$ and the mean range of refractory periods from 12.2 to $6.8 \mathrm{msec}(P<0.05)$. In an eighth group of patients, the significant variables were set to create either the greatest or least variability in refractoriness in order to determine the effects of controlling these factors. Bipolar pacing was used in this group of patients. The within-subject variance component decreased from 31.7 to $3.5(P<0.01)$ and the mean range of refractory period determinations decreased from 15.8 to $4.4 \mathrm{msec}(P<0.01)$ when the variables found to affect the reproducibility of refractoriness were set in a fashion to decrease variability. In conclusion: (1) Conventional measurement of ventricular refractoriness often is associated with variability in the range of 10-15 msec (2) Pacing at $10 \mathrm{~mA}$, unipolar rather than bipolar pacing, atrioventricular synchrony during basic drive trains, a large number of trains before refractoriness is reached, and bipolar distal cathodal pacing decrease this variability and provide greater reproducibility in the measurement of ventricular refractoriness. (PACE, Vol. 14, September 1991)

reproducibility, electrophysiological study, refractoriness

Supported in part by Grant 5M01-RR00042-25 from the National Institutes of Health, Bethesda, Maryland. Dr. Kadish is a recipient of an NIH First Award Number HL40667.

Address for reprints: Alan H. Kadish, M.D., Northwestern Memorial Hospital, Section of Cardiology, Wesley Pavilion, 250 East Superior Street, Suite 524, Chicago, IL 60611. Fax: (312) 908-6003.

Received March 8, 1991; revision May 22, 1991; accepted May 23, 1991.

\section{Introduction}

When measuring ventricular refractoriness during clinical electrophysiological studies, determinations may be repeated once for verification. Although studies have examined the influence of a variety of factors on refractoriness, ${ }^{1-9}$ the inherent reproducibility of such measurements has never been carefully examined. The goal of 
this study was to quantitate the reproducibility of clinical measurements of ventricular refractoriness and to determine what factors may affect this reproducibility.

\section{Methods}

\section{Study Design}

This study was designed to determine the effects of the seven variables shown in Table I on the reproducibility of measurements of the right ventricular effective refractory period (VERP). These variables were felt to represent factors that might possibly affect the measurement of ventricular refractoriness and that could be controlled. Each variable had two conditions (1 and 2). The effects of individual variables were tested by fixing six of the seven variables in condition 1 and performing ten consecutive measurements of refractoriness with the variable being examined in condition 1 and condition 2. For example, to test autonomic effects, all other variables were set in condition 1 and refractoriness was determined ten times before and ten times after the administration of atropine and propranolol. Seven groups of patients were studied so that each variable could be tested individually. In addition, after the effects of the tested variables on the variability in ventricular refractoriness were determined, measurements of refractoriness were repeated ten times in an eighth group of patients with all the variables set in the condition in which there was the least reproducibility and compared to the condition in which there was greatest reproducibility.

\section{Patient Population}

Eighty patients undergoing a clinically-indicated electrophysiological test were the subjects of this study. Patients were off all antiarrhythmic medications for at least five half-lives at the time of the electrophysiological test. Patients who had arrhythmias induced with a single ventricular extrastimulus, who were in atrial fibrillation, or who had sinus tachycardia with rates faster than 110 beats/min were excluded from the study. A selection criterion for the groups in which the effect of the atrioventricular (AV) relationship was tested (group 6 and 8) was the presence of ventriculoatrial (VA) dissociation during ventricular pacing at a cycle length of $500 \mathrm{msec}$. Patients with a contraindication to intravenous propranolol or atropine were excluded from the group of patients undergoing autonomic blockade (group 7). There were 60 men and 20 women. The mean age of the patients was $53.8 \pm 15.9$ years. Forty of the 80 patients $(50 \%)$ had underlying heart disease. Patient characteristics and indications for electrophysiological testing are shown in Table II. There were no significant differences in the presence of heart disease, the sinus cycle length or ejection fraction (available in 41 of 80 patients) between the groups.

\section{Determination of Refractoriness}

Electrophysiological studies were performed in the fasting, unsedated state, after informed consent was obtained. The research protocol conformed with the principles in the declaration of Helsinki. Two or three quadripolar catheters were

Table I.

Variables Tested

\begin{tabular}{llll}
\hline \multicolumn{1}{c}{ Variable } & \multicolumn{1}{c}{ Condition 1} & \multicolumn{1}{c}{ Condition 2 } \\
\hline 1 & Stimulation current & $2 \times$ threshold & $10 \mathrm{~mA}$ \\
2 & Train coupling & $\mathrm{S}_{1}$ delay $-500 \mathrm{msec}$ & $\mathrm{S}_{1}$ delay variable \\
3 & Intertrial pause & none & 1 minute \\
4 & Pacing configuration & Bipolar & Unipolar \\
5 & Bipolar polarity & Anode distal & Cathode distal \\
6 & AV relationship & AV synchrony & AV dissociation \\
7 & Autonomic tone & Uncontrolled & Blockade with atropine and propranolol \\
\hline
\end{tabular}

$A V=$ atrioventricular; $S_{1}$ delay $=$ coupling of the first beat of the drive to a spontaneous beat. 
Table II.

Patient Characteristics

\begin{tabular}{ll} 
Age & $53.8 \pm 15.9$ years \\
Sex & 60 Male, 20 Female \\
Heart disease & 40-None \\
& 18-Coronary artery disease \\
& 11-Dilated cardiomyopathy \\
& 4-Hypertensive heart disease \\
& 4-Valvular heart disease \\
& 2-Mitral valve prolapse \\
& 1-Hypertrophic cardiomyopathy \\
& 31-Ventricular tachycardia or \\
Indication for & fibrillation \\
electrophysio- & 28-Syncope or presyncope \\
logical study & 5-Bradycardia or heart block \\
& 16-Supraventricular tachycardia \\
\hline
\end{tabular}

advanced from the femoral vein under fluoroscopic guidance and positioned in the heart. Catheters were positioned as required for the clinical electrophysiological test. For the purposes of this protocol, a catheter in the high right atrium was used to pace the atria and a 6 French quadripolar catheter with a 1-cm interelectrode distance was used to determine ventricular refractoriness. All patients had a stable stimulation threshold of 1 $\mathrm{mA}$ or less. A Bloom DTU 210 stimulator (Bloom Associates Ltd., Reading, PA, USA) was used for pacing and data were recorded on a Siemens 7 Mingograf recording system (Siemens, Elema, Solna, Sweden). The pacing stimuli were $2 \mathrm{msec}$ in duration.

The basic drive cycle length was $500 \mathrm{msec}$ and determinations were made with trains of 8 beats, a single extrastimulus, and a 3 -second intertrain pause. Refractoriness was initially determined by decreasing the coupling interval of the extrastimulus $\left(\mathrm{S}_{2}\right)$ by $10-\mathrm{msec}$ steps, beginning at $290 \mathrm{msec}$, until $S_{2}$ failed to capture. The coupling interval was increased by $10 \mathrm{msec}$ and then decreased in 2-msec steps until $S_{2}$ failed to capture. The VERP was defined as this $S_{1}-S_{2}$ coupling interval.

\section{Testing of Variables}

Eight groups of ten patients each were studied. In groups 1-7, one of the variables in Table I was tested in condition 1 and condition 2 . Re- fractoriness was measured ten times in each condition. Conditions were tested sequentially with ten determinations made under one condition and ten under the second condition. In each group of ten patients except group 7 , condition 1 was tested first in five of the patients and condition 2 was tested first in the other five patients. After the variables that had a significant effect on the variability of refractoriness were determined in groups 1-7, ten additional patients (group 8) had ten determinations of refractoriness made under two conditions: all variables set to produce maximal variability (condition 1) and all variables set to decrease variability (condition 2). This allowed a determination of the magnitude of variability produced by all the variables.

Variable 1: The diastolic threshold was determined with an accuracy of $0.1 \mathrm{~mA}$ at a pacing cycle length of $500 \mathrm{msec}$. A current strength of twice diastolic threshold was used as condition 1 and $10 \mathrm{~mA}$ as condition 2 .

Variable 2: Under condition 1, the first beat of each basic drive train was coupled to the last spontaneous beat at a fixed interval of $500 \mathrm{msec}$. This insured that all 8 beats of the basic drive train captured the ventricle. In condition 2, there was no synchronization of the basic drive train to spontaneous beats. Thus, a variable number of basic train beats may have failed to capture.

Variable 3: In condition 1, patients were paced for 1 minute with 8-beat basic drive trains and 3-second intertrain pauses before the start of refractoriness determinations. After refractoriness was reached, the coupling interval was increased by $10 \mathrm{msec}$ from VERP and another trial performed. There was no interruption in the sequence of 8-beat trains separated by 3 -second pauses. In condition 2 , pacing was stopped for at least 1 minute between determinations of refractoriness. Determinations of refractoriness were started alternately at $S_{1}-S_{2}$ coupling intervals of 10 or $40 \mathrm{msec}$ longer than the previous VERP. This created a variable number of $S_{1}$ trains before refractoriness was reached.

Variable 4: Bipolar pacing with the anode as the distal pole was used as condition 1. Unipolar cathodal pacing was used in condition 2 .

Variable 5: All trials were performed with bipolar pacing using the two distal poles of the cath- 
eter. In the condition 1, the anode was used as the distal pole; whereas, in condition 2 the cathode was the distal pole.

Variable 6: All patients in this group had VA dissociation during basic drive. The atria and ventricles were paced simultaneously in condition 1 and atrial activation was not controlled in condition 2.

Variable 7: Determinations were made before (condition 1) and after (condition 2) the administration of intravenous propranolol $(0.2 \mathrm{mg} / \mathrm{kg}$ at $1-2 \mathrm{mg} / \mathrm{min})$ and atropine $(0.04 \mathrm{mg} / \mathrm{kg}$ at $1 \mathrm{mg} /$ $\mathrm{min}$ ). The mean propranolol dose was $14.4 \pm 1.6$ $\mathrm{mg}$ and the mean atropine dose was $2.9 \pm 0.4 \mathrm{mg}$. These doses of propranolol and atropine have been shown to inhibit the effects of the beta adrenergic and parasympathetic stimulation on refractoriness. ${ }^{7.9}$

\section{Data Analysis}

All data are expressed as mean \pm standard deviation. Mean values for ventricular refractoriness under each condition were calculated based on 100 determinations (ten in each patient). Differences in absolute values of refractoriness for each set of trials were determined using a repeated measures analysis of variance. For each group of patients, under each of the two conditions, a mixed model analysis of variance was run to determine the between-subject and within-subject variance components. The square root of the within-subject variance component divided by the square root of the sample size equals one standard deviation of the mean. The within-subject variance component was used as an index for the variability of refractoriness. ${ }^{10}$ This within-subject variance component was then compared between the two conditions within a trial using Snedecor's F-test. ${ }^{10}$ The range in determination of refractoriness was defined as the difference between the longest and shortest value of the ten determinations in each patient under each condition. Thus, this difference was calculated for each variable under each condition and compared using a paired t-test. However, the primary method used to decide whether a variable had an effect on the reproducibility of VERP was the within-subject variance component.

\section{Results}

\section{Thresholds and Magnitude of VERP}

The mean and standard deviation of the VERP determinations for each of the seven groups under the two conditions are shown in Table III. Of the seven variables tested, only the strength of the stimulation current (twice diastolic vs $10 \mathrm{~mA}$ ) significantly affected the magnitude of ventricular refractoriness. At twice diastolic threshold, the mean VERP was $247.3 \pm 12.9$ msec versus 219.8

Table III.

Ventricular Refractory Period

\begin{tabular}{|c|c|c|c|c|c|}
\hline \multirow[b]{2}{*}{ Group } & \multirow[b]{2}{*}{ Variable } & \multicolumn{2}{|c|}{ Condition 1} & \multicolumn{2}{|c|}{ Condition 2} \\
\hline & & $\begin{array}{c}\text { Mean } \\
\text { (msec) }\end{array}$ & $\begin{array}{c}\text { SD } \\
\text { (msec) }\end{array}$ & $\begin{array}{c}\text { Mean } \\
\text { (msec) }\end{array}$ & $\begin{array}{c}\text { SD } \\
\text { (msec) }\end{array}$ \\
\hline 1 & Stimulation current & 247.3 & 12.9 & $219.8^{+}$ & 15.6 \\
\hline 2 & Train coupling & 235.7 & 15.5 & 237.7 & 14.2 \\
\hline 3 & Intertrial pause & 231.5 & 25.4 & 232.5 & 27.5 \\
\hline 4 & Pacing configuration & 251.0 & 18.7 & $243.8^{\#}$ & 24.0 \\
\hline 5 & Bipolar polarity & 252.7 & 21.3 & 251.8 & 22.0 \\
\hline 6 & AV relationship & 231.6 & 22.5 & 232.6 & 22.5 \\
\hline 7 & Autonomic tone & 231.8 & 16.1 & 226.9 & 16.5 \\
\hline 8 & Multiple & 259.6 & 16.4 & $218.6^{+}$ & 13.9 \\
\hline
\end{tabular}


$\pm 15.6 \mathrm{msec}$ at $10 \mathrm{~mA}(\mathrm{P}<0.01)$. There was a trend for unipolar pacing to yield a shorter VERP than bipolar pacing (group 4), but this difference did not reach significance $(P=0.06)$.

Differences in pacing threshold were observed when comparing bipolar to unipolar pacing and when comparing bipolar configurations (distal anode vs distal cathode). The mean bipolar pacing threshold was $0.5 \pm .2 \mathrm{~mA}$ versus $0.9 \pm$ $0.2 \mathrm{~mA}$ for unipolar pacing $(\mathrm{P}<0.001$; group 4$)$. The mean pacing threshold was $0.6 \pm 0.2 \mathrm{~mA}$ for bipolar pacing with a distal cathode versus $0.4 \pm$ 0.1 for bipolar pacing with a distal anode $(\mathrm{P}<$ 0.01 ; group 5).

\section{Variables Affecting the Reproducibility of Refractoriness}

Five variables significantly affected the reproducibility of measurements of ventricular refractoriness. Table IV shows the mean within-subject variance component for each variable under each condition. Pacing at twice threshold (group 1), the use of a variable pause before beginning the next series of drive trains (group 3), bipolar pacing (group 4), the use of the distal pole as the anode during bipolar pacing (group 5), and VA dissociation during the basic drive trains (group 6) all created a greater degree of variability in serial determinations of VERP than the other corresponding condition (Table I). There was no interaction be-

Table IV.

Variability in VERP as Measured by Within-Subject Variance Components

\begin{tabular}{clcc}
\hline Group & \multicolumn{1}{c}{ Variable } & $\begin{array}{c}\text { Condition } \\
\mathbf{1}\end{array}$ & $\begin{array}{c}\text { Condition } \\
\mathbf{2}\end{array}$ \\
\hline 1 & Stimulation current & 10.1 & $4.5^{\star \star}$ \\
2 & Train coupling & 6.8 & 4.9 \\
3 & Intertrial pause & 3.3 & $16.5^{\star \star}$ \\
4 & Pacing configuration & 6.4 & $4.4^{\star}$ \\
5 & Bipolar polarity & 9.4 & $3.3^{\star \star}$ \\
6 & AV relationship & 5.7 & $21.2^{\star \star}$ \\
7 & Autonomic tone & 6.3 & 7.6 \\
8 & Multiple & 31.7 & $3.5^{\star \star}$ \\
\hline
\end{tabular}

" $P<0.05 ;{ }^{* *} P<0.001$, all comparisons versus Condition 1; VERP $=$ ventricular effective refractory period. tween the presence of heart disease and variability in the determination of refractoriness.

Pacing at $10 \mathrm{~mA}$ was associated with less variability in the measurement of refractoriness than pacing at twice threshold. When all other variables were held constant, the within-subject variance component was 10.1 for pacing at twice threshold and 4.5 when pacing at $10 \mathrm{~mA}(\mathrm{P}<$ 0.001 ). Differences between the longest and shortest measurements of VERP are shown in Table V. The mean range of refractory periods was $8.6 \mathrm{msec}$ at twice threshold and $6.2 \mathrm{msec}$ at $10 \mathrm{~mA}$, but the difference in range of VERP determinations did not reach significance.

With all other variables held constant, the within-subject variance component in the measurement of VERP for continuous pacing between determinations was 3.3 versus $16.5(\mathrm{P}<0.001)$ for determinations with a variable pause (group 3 , Table IV). When continuous pacing was used, there was a mean range of $4.8 \mathrm{msec}$ (Table V). This range increased to $10.8 \mathrm{msec}$ when a pause was used between determinations; however, this difference did not reach significance.

Unipolar pacing was associated with a lower within-subject variance component (4.4) than bipolar pacing $(6.3 ; \mathrm{P}<0.05$; Table IV). The mean range of refractory periods was $6.4 \mathrm{msec}$ for bipolar pacing and $5.8 \mathrm{msec}$ for unipolar pacing (Table V).

During bipolar pacing, use of the distal pole as the cathode was associated with less variability (mean within-subject variance component 5.7) than using the distal pole as the cathode mean variance component (21.2; $\mathrm{P}<0.001$; Table IV). The mean range of VERP determinations was also smaller using distal cathodal pacing (Table V), but this difference did not reach significance.

Comparing the within-subject variance component in the presence and absence of AV synchrony (group 6) demonstrated a greater variability when the AV relationship was not controlled (Table IV). The presence of AV synchrony decreased the range of refractory periods from a mean of 12.2 to $6.8 \mathrm{msec}(\mathrm{P}<0.05$; Table $\mathrm{V})$.

\section{Maximum Variability in Refractoriness}

To determine the maximum variability in the determination of the VERP, the variables that were shown to affect the reproducibility of the VERP 


\begin{tabular}{|c|c|c|c|c|c|}
\hline \multirow[b]{3}{*}{ Group } & \multicolumn{5}{|c|}{$\begin{array}{c}\text { Table V. } \\
\text { Differences Between Shortest and Longest VERP }\end{array}$} \\
\hline & & \multicolumn{2}{|c|}{ Condition 1} & \multicolumn{2}{|c|}{ Condition 2} \\
\hline & & Mean & SD & Mean & SD \\
\hline 1 & Stimulation current & 8.6 & 4.5 & 6.2 & 1.5 \\
\hline 2 & Train coupling & 7.0 & 3.7 & 5.6 & 4.8 \\
\hline 3 & Intertrial pause & 4.8 & 2.1 & 10.8 & 6.1 \\
\hline 4 & Pacing configuration & 6.4 & 4.3 & 5.8 & 2.7 \\
\hline 5 & Bipolar polarity & 7.6 & 4.8 & 5.0 & 1.9 \\
\hline 6 & AV relationship & 6.8 & 3.8 & $12.2^{*}$ & 5.9 \\
\hline 7 & Autonomic tone & 7.0 & 2.8 & 7.0 & 4.1 \\
\hline 8 & Multiple & 15.8 & 9.4 & $4.4^{* *}$ & 3.1 \\
\hline
\end{tabular}

were set in a fashion to maximize this variability (pause between trials, AV asynchrony, stimulus intensity of twice diastolic threshold, and bipolar pacing with the anode as the distal pole). It was not possible to evaluate unipolar pacing and the bipolar configuration in the same trial and thus, bipolar pacing was chosen to reflect conventional techniques. Variables were set to maximize variability in condition 1 and to minimize variability in condition 2. Thus, condition 1 consisted of bipolar distal anodal pacing at twice threshold without atrial pacing and with a variable pause between trials. Controlling these variables in condition 2 with atrial pacing at $10 \mathrm{~mA}$, bipolar distal cathodal pacing and continuous pacing between trials decreased the within-subject component of variance from 31.7 to $3.5(\mathrm{P}<0.001)$ and the mean range of refractory periods from 15.8 to $4.4 \mathrm{msec}$ $(\mathrm{P}<0.01)$.

\section{Discussion}

Seven variables were examined to determine if they affect the reproducibility of VERP determinations. Pacing at $10 \mathrm{~mA}$, the presence of $\mathrm{AV}$ synchrony during basic drive trains, continuous pacing between determinations, unipolar pacing, and the use of the cathode as the distal pole in bipolar pacing were found to decrease variability in serial determinations of refractoriness. When these variables were set in a fashion to maximize variability (group 8), up to a 40 -msec difference between the shortest and longest values (mean $15.8 \mathrm{msec}$ ) was found on serial determinations of refractoriness. Controlling these variables decreased the within-subject component of variance by approximately $90 \%$ and the range of refractory periods by $72 \%$, indicating that the factors that are responsible for most of the variability in determination of ventricular refractoriness by the catheter technique were identified in this study.

\section{AV Synchrony During Basic Drive Trains}

Hemodynamic factors are known to affect ventricular refractoriness. ${ }^{8.11}$ This phenomenon, referred to as contraction-excitation feedback, may operate in resting and actively contracting muscle. ${ }^{11-13}$ Several clinical studies have recently confirmed the presence of contraction-excitation feedback in patients undergoing pulmonary valvuloplasty and in patients after cardiopulmonary bypass. ${ }^{14-15}$ Since the timing of atrial systole can affect ventricular size, ${ }^{16,17}$ it might be expected to alter ventricular refractoriness. We have recently shown that ventricular refractoriness is shorter when measured during AV simultaneous pacing than when measured during pacing at an AV interval of 0 msec. $^{18}$ Since differing paced AV relationships alter refractoriness, we found that variability in VERP determination was increased when the timing of atrial systole was not controlled. 


\section{Continuous Pacing}

The adaptation of ventricular refractoriness to a change in rate is a complex phenomenon that has been extensively studied. ${ }^{19-21}$ As heart rate increases, action potential duration and ventricular refractoriness shorten in a time-dependent fashion. Although some shortening occurs within the first few beats, steady state changes may take up to several minutes to develop. ${ }^{22}$ In routine clinical and some experimental electrophysiological studies, 8-beat trains are separated by a pause, but it has not been clear if the pause is of adequate length to restore refractoriness to steady-state conditions at the longer prepacing cycle length. It is likely that as the number of trains is increased, a gradual decrease in refractoriness is manifest. Thus the number of 8-beat trains prior to reaching refractoriness may affect its determination. We compared the variability in refractoriness after a large number of trains (1 min of conditioning and continuous pacing between trials) to that in which the number of trains prior to reaching refractoriness varied and found that the continuous pacing method was subject to far less variability.

\section{Bipolar Polarity}

When the distal pacing electrode was the cathode, less variability in the measurement of ventricular refractoriness was noted. Several possible explanations exist for this difference. It has been shown in a previous study that the anode as well as the cathode may contribute to ventricular excitation during pacing. ${ }^{23}$ However, the extent of each pole's contribution in individual cases is unknown and may depend on a variety of factors including contact of the proximal pole with the myocardium. If the cathodal contribution to pacing is routinely larger and its position is varying because it is not in direct contact with the myocardium, then a greater variability in refractoriness might be noted. Another possibility is that an early diastolic dip in the strength-interval excitability relationship has been noted during anodal and less frequently bipolar stimulation in experimental and clinical studies. ${ }^{24,25}$ If this dip is present, small changes in the location of the dip may increase the variability in refractoriness determined at twice threshold. Since the dip is a phenomenon of anodal rather than cathodal pacing, it may be present more frequently during bipolar pacing with a distal anode.

\section{Coupling of Basic Drive Trains to Spontaneous Rhythm}

The number of beats in a drive train will affect the value of ventricular refractoriness because of the gradual adaptation of refractoriness to changes in cycle length, as noted above. ${ }^{22}$ Failure to couple the first beat of a drive train to spontaneous beats may result in lack of capture of the first beats of the train, in effect shortening the length of the train and thereby affecting refractoriness. We thus evaluated whether altering train coupling from a fixed to variable amount would affect reproducibility measurements. A trend towards greater reproducibility when the first beat of the train was fixed coupled was noted but it did not reach statistical significance, perhaps because the differences in the effective train length were minimal.

\section{Stimulation Current}

The relationship between pacing current and refractoriness is described by a curvilinear relationship referred to as the strength-interval curve ${ }^{6,26}$ Refractoriness decreases with increasing current until a plateau is reached. A stimulus intensity of twice diastolic threshold is generally on the ascending portion of the strength-interval curve whereas $10 \mathrm{~mA}$ stimulation is on the plateau. If slight variations in the shape of the strength-interval curve occur this might alter refractoriness at twice threshold far more than at 10 $\mathrm{mA}$. Refractoriness determined at $10 \mathrm{~mA}$ was in fact subject to less variability, suggesting that such small changes in the shape or position of the strength-interval curve may occur. In addition, microdislodgment of the electrode catheter would be expected to have less impact at a current strength of $10 \mathrm{~mA}$ than at twice threshold.

\section{Unipolar Versus Bipolar Stimulation}

Although bipolar stimulation (positive and negative poles as the distal catheter poles) has been most commonly used in electrophysiological studies, a comparison has shown that unipolar pacing is equally effective in inducing arrhythmias. ${ }^{27}$ Unipolar stimulation should be less subject to proximal pole motion and thus could po- 
tentially provide a less variable refractory period. A small difference in the variability of unipolar and bipolar refractory periods was found, suggesting that proximal pole motion may affect the variability in refractory period measurements.

\section{Autonomic Blockade}

Variations in autonomic tone may affect refractoriness. $^{3,7,9}$ Beta adrenergic stimulation shortens action potential duration and refractoriness. ${ }^{28,29}$ Vagal stimulation has been shown experimentally and clinically to lengthen ventricular refractoriness and alpha stimulation produces a small lengthening as well. ${ }^{7,9} \mathrm{We}$ found no effect of autonomic blockade on ventricular refractoriness suggesting that any spontaneous short-term variations in autonomic tone are too small to affect refractoriness.

\section{Other Factors}

Approximately $90 \%$ of the variability in repeated measures of refractoriness found in this study (as defined by the within-subject variance component) can be attributed to the factors we have identified.

Measurements performed in group 8 patients and condition 1 represent the maximum degree of variability present, whereas those in group 8 and condition 2 (bipolar distal cathodal pacing at 10 $\mathrm{mA}$, atrial pacing, and continuous pacing between trials) represent the minimum variability. Controlling these factors decreased the within-subject component of variance from 31.7 to 3.5 and the mean difference between shortest and longest determination from 15.8 to $4.4 \mathrm{msec}$. Microdisplacement of the catheter tip is an unquantifiable possible cause for some of the small remaining variability. Natural biological variability or other unknown factors may be responsible for some of the small variability as well.

\section{Limitations}

Alpha adrenergic antagonists have been found to have small but measurable effects on ventricular refractoriness. Because of the constraints of a clinical study, alpha blockade was not administered as part of the autonomic blockade protocol and thus variability in refractoriness secondary to changing alpha adrenergic tone cannot be excluded with certainty. However, the magnitude of the effect of the alpha adrenergic system on ventricular refractoriness is small compared to that of the other components of the autonomic nervous system. ${ }^{7}$

Another limitation is that determination of ventricular refractoriness using an incremental method was not compared to the decremental method in this study. It is possible that by eliminating the conditioning effect of the capture of extrastimuli that occurs during the decremental method, the variability in determination of refractoriness may decrease. However, since one of the variables evaluated in this study was a conditioning period of more than 1 minute and because any conditioning effect of the capture of a premature stimulus should be constant during serial determinations of refractoriness, it is not likely that incremental and decremental methods would yield differences in the variability in the determination of refractoriness.

\section{Implications}

The determination of ventricular refractoriness has been used to guide coupling intervals during programmed stimulation, to define drug effects, to examine the pathophysiology of arrhythmias, and to predict drug efficacy. ${ }^{30-32}$ Using a conventional protocol that includes stimulation at twice threshold and that does not include AV simultaneous pacing, a conditioning period, or continuous drive trains, a mean difference of 10-15 msec was found on repeated determinations of ventricular refractory periods. Controlling these factors using AV simultaneous pacing, a stimulus intensity of $10 \mathrm{~mA}$, bipolar cathodal pacing, a 3minute conditioning period (of an 8-beat train followed by a pause), and continuous drive trains will decrease the mean difference to $<5 \mathrm{msec}$ and improve reliability of refractory period measurements.

Acknowledgments: The authors would like to thank Keith Childs for his research assistance and Veronica Jefferson for typing.

\section{References}

1. Goodman DJ, Rosen RM, Rider AK, et al. The effect of cycle length on cardiac refractory periods in the 
denervated human heart. Am Heart J 1976; 91:332-338.

2. Denes $\mathrm{P}, \mathrm{Wu} \mathrm{D}$, Dhingra $\mathrm{R}$, et al. Effect of cycle length on cardiac refractory periods. Circulation $1974 ; 49: 32-41$.

3. Guss SB, Kastor JA, Josephson ME, et al. Human ventricular refractoriness: Effects of cycle length, pacing site and atropine. Circulation 1976; 53:450-455.

4. Weiner I, Kunkes S, Rubin D, et al. Effects of sudden change in cycle length on human atrial, atrioventricular nodal and ventricular refractory periods. Circulation 1981; 64:245-248.

5. Kastor JA, Josephson ME, Guss SB, et al. Human ventricular refractoriness. II. Effects of procainamide. Circulation 1977; 56:462-467.

6. Greenspan AM, Camardo JS, Horowitz LN, et al. Human Ventricular Refractoriness: Effects of increasing current. Am J Cardiol 1981; 47:244-250.

7. Prystowsky EM, Jackman WM, Rinkenberger RL, et al. Effect of autonomic blockade on ventricular refractoriness and atrioventricular nodal conduction in humans. Circ Res 1981; 49:511-520.

8. Gornick CC, Tobler HG, Tuna IC, et al. Electrophysiologic effects of left ventricular free wall traction in intact heart. (abstract) PACE 1986; 9:275.

9. Morady F, Kou WH, Nelson SD, et al. Accentuated antagonism between B-adrenergic and vagal effects on ventricular refractoriness in humans. Circulation 1988; 77:289-297.

10. Milliken GA, Johnson DE. Analysis of Messy Data. Volume 1: Designated Experiments. New York, Van Nostrand Reinhold. 1984, pp. 246-262.

11. Lab MJ. Contraction-excitation feedback in myocardium physiological basis and clinical relevance. Circ Res 1982; 50:757-766.

12. Lab MJ, Allen CH. The effects of shortening on myoplasmic calcium concentration and on the action potential in mammalian ventricular muscle. Circ Res $1984 ; 55: 825-829$.

13. Franz MR, Burkhoff D, Yue DT. Mechano-electrical feedback in the intact isolated perfused canine heart. (abstract) Circulation 1985; 72:III-382.

14. Levine JH, Guarnieri R, Kadish AH, et al. Changes in myocardial repolarization in patients undergoing balloon valvuloplasty for congenital pulmonary stenosis: Evidence for contraction-excitation feedback in humans. Circulation 1988; 77:70-77.

15. Taggart P, Sutton PMI, Treasure T, et al. Monophasic action potentials at discontinuation of cardiopulmonary bypass: Evidence for contractionexcitation feedback in man. Circulation 1988; $77: 1266-1275$.

16. Gilmore JP, Sarnoff SJ, Mitchell JH, et al. Synchronicity of ventricular contraction: Observations comparing hemodynamic effects of atrial and ventricular pacing. Br Heart J 1963; 28:299-307.

17. Romero LR, Haffajee CI, Levin W, et al. Non-invasive evaluation of ventricular function and vol- umes during atrioventricular sequential and ventricular pacing. PACE 1984; 7:10-17.

18. Kadish AH, Kou WH, Schmaltz S, et al. Effect of atrioventricular relationship on ventricular refractoriness in humans. Am J Physiol 1990; H1463$\mathrm{H} 1470$.

19. Mendez C, Grubzit CC, Moe GK. Influence of cycle length upon the refractory period of auricles, ventricles and A-V node in the dog. Am J Physiol 1956 184:287-295.

20. Carmeliet E. Repolarization and frequency in cardiac cells. J Physiol (Paris) 1977; 73:903-923.

21. Boyett MR, Jewell BR. Analysis of the effects of changes in rate and rhythm upon electrical activity in the heart. Prog Biophys Mol Biol 1980; 36:152.

22. Brownstein SL, Bauernfeind R, Welch $W$, et al. Cumulative effects of cycle length on ventricular refractoriness in man. (abstract) Pace 1987; $10: 432$.

23. Stevenson WG, Weiner I, Weiss JN. Contribution of the anode to ventricular excitation during bipolar programmed electrical stimulation. Am J Cardiol 1986; 57:582-586.

24. Van Dam RT, Durrer D, Strackee J, et al. The excitability cycle of the dog's left ventricle determined by anodal, cathodal, and bipolar stimulation. Circ Res 1956; 4:196-202.

25. Mehra R, Furman S. Comparison of cathodal, anodal and bipolar strength-interval curves with temporary and permanent pacing electrodes. $\mathrm{Br}$ Heart J 1979; 41:468-476.

26. Camardo JS, Greenspan AM, Horowitz LN, et al. Strength-interval relation in the human ventricle. Effect of procainamide. Am J Cardiol 1980; 45:856-860.

27. Stevenson WG, Weine RRI, Weiss JN. Comparison of bipolar versus unipolar programmed stimulation for the initiation of ventricular arrhythmias. Circulation 1986; 73:693-700.

28. Freedman RA, Swerdlow DS, Echt RA, et al. Facilitation of ventricular tachyarrhythmia induction by isoproterenol. Am J Cardiol 1984; 54:765770.

29. Reddy CP, Gettes LS. Use of isoproterenol as an aid to electric induction of chronic recurrent ventricular tachycardia. Am J Cardiol 1979; 44:705713.

30. Mason J, Winkle R. Electrode-catheter arrhythmia induction in the selection and assessment of antiarrhythmic drug therapy for recurrent ventricular tachycardia. Circulation 1978; 58:971-985.

31. Cain ME, Martin TC, Marchlinski FE, et al. Changes in ventricular refractoriness after an extrastimulus: Effects of prematurity, cycle length and procainamide. Am J Cardiol 1983; 52:9961001.

32. Vassallo JA, Cassidy DM, Kindwall E, et al. Nonuniform recovery of excitability in the left ventricle. Circulation 1988; 78:1365-1372. 
This document is a scanned copy of a printed document. No warranty is given about the accuracy of the copy. Users should refer to the original published version of the material. 